\title{
Proposal to Study Uniqueness Problem of Rayleigh Wave in Half-Space Kelvin Viscoelastic Media
}

\author{
Hua Cheng', Takashi Takiguchi ${ }^{2}$ \\ ${ }^{1}$ Department of Aeronautics and Astronautics, Fudan University, Shanghai, China \\ ${ }^{2}$ Department of Mathematics, National Defense Academy, Yokosuka, Kanagawa-Pref, Japan \\ Email: huacheng@fudan.edu.cn
}

How to cite this paper: Cheng, H. and Takiguchi, T. (2019) Proposal to Study Uniqueness Problem of Rayleigh Wave in Half-Space Kelvin Viscoelastic Media. Journal of Applied Mathematics and Physics, 7 , 2074-2088.

https://doi.org/10.4236/jamp.2019.79142

Received: July 28, 2019

Accepted: September 21, 2019

Published: September 24, 2019

Copyright () 2019 by author(s) and Scientific Research Publishing Inc. This work is licensed under the Creative Commons Attribution International License (CC BY 4.0).

http://creativecommons.org/licenses/by/4.0/

\begin{abstract}
Rayleigh wave is an interference wave of longitudinal wave and transverse wave which propagate along the free surface of solids. There remains a dispute about the number of Rayleigh waves in viscoelastic media until now, which is an essential problem of Rayleigh wave propagation. The purpose of this study is to propose a brief way of handling this essential problem within half-space Kelvin viscoelastic media. Starting from the dynamic equations of transverse wave and longitudinal wave based on Kelvin viscoelastic model, this study sets the complex wave number as a variable, introduces complex moduli and complex exponential factors, then a characteristic equation of Kelvin viscoelastic Rayleigh wave in half space is derived and simplified support for analysis of its uniqueness. After reviewing mathematical models describing phenomena of having multiple solutions but uniqueness when a natural condition is taken into account, a conjecture is given that the Rayleigh wave in Kelvin viscoelastic media must be unique if we assume a natural condition in accordance with the natural phenomena.
\end{abstract}

\section{Keywords}

Rayleigh Wave, Viscoelastic, Wave Equation, Characteristic Equation, Kelvin Model

\section{Introduction}

The Rayleigh wave is a surface wave that propagates along the surface of a medium [1]. It has a wide application prospect in many research aspects, ranging from seismic wave, surface sound wave and material science etc. In early seismic 
exploration, Rayleigh wave was once treated as disturbing wave until the $50 \mathrm{~s}$ of last century when the frequency dispersion characteristics had been confirmed mathematically [2] [3], its application value has been gradually excavated. Rayleigh wave is essentially the formation of interference on the surface of the medium of two kinds of body waves, which are the longitudinal wave (P-wave) and the transverse wave (S-wave) propagating inside the medium. Therefore, Rayleigh (1885) [1] summed it up as the simultaneous solution of the equation of transverse wave and longitudinal wave. Yet to this day, the method of the characteristic equation of surface wave velocity he deduced is still the important way to seek for Rayleigh wave.

A viscoelastic medium, which is dissipative, is a state between elastic solid and viscous fluid, showing both the elastic and viscous properties at the same time. When considering that the propagation process of Rayleigh wave will result in some attenuation, compared with an ideal elastic medium, a viscoelastic medium is closer to the actual condition of the engineering. Study of Rayleigh wave in viscoelastic media was first discussed by Scholte [4], he proofed that the Rayleigh wave also existed on the surface of half-space viscoelastic media, but he was not sure whether the Rayleigh wave he calculated was the only one valid. In 1960, from the mathematical aspect of the effective characteristic root, Bland [5] raised the question of the existence and uniqueness of Rayleigh wave in viscoelastic media "It has not yet been shown that for any viscoelastic material there is one and only one such root" [[5], p. 75]. In response to this question, after a series of studies based on linear viscoelastic models, Currie et al. [6] [7] [8] came to a conclusion in 1977 that there was more than one possible Rayleigh wave in the viscoelastic half-space surface. In most cases, there existed two while in some special material there would even be three possible Rayleigh waves [7]. What's more, they predicted that the velocity of viscoelastic surface wave would sometimes be higher than that of the body wave, and there would even be retrograde wave [6] [7]. After that, Bland's question seemed to be solved. But, on the one hand, there is no report about the practical examples that the velocity of surface wave will surpass the body wave velocity nor the retrograde propagation phenomenon. On the other hand, in 2001 Romeo [9] [10] promoted Nkemzi's elastic Rayleigh wave equation [11] to linear viscoelastic situation, thus put forward an opposite viewpoint. He took advantage of complex modulus to analyze the root number of the characteristic equation of viscoelastic half-space Rayleigh wave. Although his derivation process contained multiple variable substitutions, which appeared complicated and obscure, he deemed that there is only one complex root valid, while the other two are invalid. This implied that there is only one truly valid Rayleigh wave in viscoelastic half-space surface. Obviously, Romeo's opinion denied the conclusion by Currie et al., but it hasn't been widely accepted yet. Except that Ivanov and Savova's study [12] supports Romeo's opinion only in the sense of wavelength fixed condition, studies in the common sense of frequency fixed condition [13] [14] and more researchers' studies [15] 
[16] [17] are in favor of the conclusion of Currie et al. The possibility of the existence of a retrograde Rayleigh surface wave is even suggested [17], and there maybe third types of Rayleigh waves in the case of some special combinations of material parameters [17]. What is worth noting is that the study in 2014 by Chirita et al. [17] is carried out based on Kelvin viscoelastic model, which is simple but very commonly adopted in practical engineering. It is interesting but a little unbelievable that the process of derivation, settings of variable and multiple variable substitutions by Chirita et al. in the study [17] were similar to Romeo's method, but in the conclusion, Romeo's opinion is completely negated. The obscure of mathematical derivation process in Chirita et al.'s article brings some difficulty to understanding for us, but the authors' work arouses our interest and makes us want to find out the truth.

As mentioned above, the question how many Rayleigh waves within the propagation along the surface of half-space viscoelastic media is still not so clear. In this study we refer to previous research methods based on above review, especially the literature of Chirita et al. [17], we follow their method using complex wavenumber as a variable and introducing complex modulus as the coefficients of the wave equation, but we try to use complex attenuation coefficients. Meanwhile, following their study, we adopt Kelvin viscoelastic model to derive the characteristic equation of Rayleigh wave in isotropic half-space model. But we don't adopt the methods which include multiple variable substitutions as those studies [9] [12] [13] [14] [17] in order to avoid complicated and vague substitutions of variables. We intend to use physically meaningful complex variables and complex coefficients, maintaining their utilization throughout the derivation, this makes it easy for the obtained mathematical model to contain physical meanings. We then make the characteristic equation simplified to analyze its uniqueness problem in the range of Kelvin viscoelastic medium.

The results obtained in the papers by Chirita et al. [17] and Currie et al. [6] [7] [8] claimed "a viscoelastic Rayleigh wave may propagate with a speed greater than either the $\mathrm{P}$ or $\mathrm{S}$ body-waves, whereas in the elastic case the speed is always less than that of $\mathrm{P}$ and $\mathrm{S}$ waves". Their results clearly conflict with the real natural condition that the Rayleigh wave is an interference wave of longitudinal wave and transverse wave. Referring to mathematical models made recently [18] [19] [20] [21], which have plural solutions but have unique solution under some condition in view of natural phenomena, so an assumption should be made that the Rayleigh wave speed is less than the transverse wave speed, we speculate that the Rayleigh wave in a Kelvin viscoelastic medium must be unique if we assume such a condition in accordance with the natural phenomenon.

The organization of the paper is as follows. The simplified characteristic equation of Rayleigh wave in isotropic half-space model is first described. Next, a brief way is shown to analyze the validity of characteristic roots in physical sense. Finally, the uniqueness problem is addressed, followed by discussion and future work. 


\section{Viscoelastic Wave Equation}

According to the theory of Continuum Mechanics, the dynamic equilibrium equation neglecting body force is expressed as

$$
\rho \frac{\partial^{2} u_{i}}{\partial t^{2}}=\sigma_{i j, j},
$$

confirm where $\sigma_{i j}$ is second order symmetric stress tensor, $\rho$ is the density of the medium, and $u_{i}$ is the displacement vector, $i, j=1,2,3$ denote the axis.

For a linear isotropic viscoelastic medium, the constitutive equation can be uniformly expressed as

$$
\sigma_{i j}=\lambda(t) \delta_{i j} * \mathrm{~d} u_{k, k}+\mu(t) *\left[\mathrm{~d} u_{i, j}+\mathrm{d} u_{j, i}\right],
$$

where, $\lambda(t), \mu(t)$ are Lame coefficients of viscoelastic material which depend on the time, ${ }^{*}$ denotes convolution operator, $\delta_{i j}$ is the Kronecker symbol. By submitting the relation (2) into (1), we obtain the displacement vector satisfying the dynamic equilibrium equation

$$
\rho \frac{\partial^{2} u_{i}}{\partial t^{2}}=[\lambda(t)+\mu(t)] * \mathrm{~d} u_{j, j i}+\mu(t) * \mathrm{~d} u_{i, j j} .
$$

As for constitutive relations of linear viscoelastic medium, there are exactly many mathematical models. In this paper, we adopt Kelvin model, which is a simplified model more efficient for describing the attenuation of elastic waves. It is also called Voigt model or Kelvin-Meyer-Voigt model [[22], p. 99] and it is commonly used in practical engineering, especially in the field of seismic exploration.

Under the assumption of the Kelvin model, the above equation of displacement vector can then be expressed as

$$
\rho \frac{\partial^{2} u_{i}}{\partial t^{2}}=\left[(\lambda+\mu)+\left(\lambda^{\prime}+\mu^{\prime}\right) \frac{\partial}{\partial t}\right] u_{j, j i}+\left(\mu+\mu^{\prime} \frac{\partial}{\partial t}\right) u_{i, j j},
$$

where, $\lambda$ and $\mu$ are the elastic partition of Lame coefficients, while $\lambda^{\prime}$ and $\mu^{\prime}$ describe two variables of the viscous partition which is independent on time, whose effect is corresponding to the $\lambda$ and $\mu$ in describing elastic medium.

Applying Helmholtz decomposition theorem on displacement vector field

$$
u_{i}=\Phi_{, i}+e_{i j k} \Psi_{k, j}, \Psi_{k, k}=0
$$

where, $\Phi$ is the scalar potential function, $\Psi_{k}$ is the vector potential function, $k=1,2,3$ denote the coordinate axes. Substitute it in (4), change the differential order, the longitudinal wave equation and the transverse wave equation can be obtained in expression of potential functions

$$
\left\{\begin{array}{l}
\rho \frac{\partial^{2} \Phi}{\partial t^{2}}=(\lambda+2 \mu) \Phi_{, j j}+\left(\lambda^{\prime}+2 \mu^{\prime}\right)\left(\frac{\partial \Phi}{\partial t}\right)_{, j j} \\
\rho \frac{\partial^{2} \Psi_{k}}{\partial t^{2}}=\mu \Psi_{k, j j}+\mu^{\prime}\left(\frac{\partial \Psi_{k}}{\partial t}\right)_{, j j}
\end{array} .\right.
$$


The above Equation (5) shows that, in Kelvin viscoelastic medium, there are also two kinds of independent wave modes like in the elastic medium, namely, the $\mathrm{P}$-wave and the S-wave. But we should pay attention to the addition of first order partial derivative term for time in their wave equations.

\section{The Characteristic Equation for Viscoelastic Medium Rayleigh Wave}

As shown in Figure 1, the lower half space is characterized by Kelvin viscoelastic medium, whose elastic coefficients are $\lambda$ and $\mu$. Its density is $\rho$ and the viscoelastic coefficients are $\lambda^{\prime}$ and $\mu^{\prime}$. The origin $O$ of the axis is located on the free surface of the half space, with the axis $O x$ on the free surface and the vertically downward axis $O z$ pointing to the inner medium part. Assume the propagation direction of the wave pointing $x$ along the plane $x z$, so the displacement component $u_{x}, u_{z}$ are independent on $y$ and $u_{y}=0$ so that it could be considered as a plane problem. The vibrations of particles happen only in plane $X Z$ and the potential function of the displacement vector in space is independent on $y$.

Since the formation of Rayleigh wave propagating on the free surface is due to the interference and superposition of the transverse wave and longitudinal wave, thus the problem comes down to establishing the simultaneous solution of the $\mathrm{P}$-wave equation and the S-wave equation, meanwhile finding the solution that the free surface boundary condition equation is satisfied. Here as Figure 1 shows, to be specifical, that the wave Equation (5) can be expressed as

$$
\left\{\begin{array}{l}
\rho \frac{\partial^{2} \Phi}{\partial t^{2}}=(\lambda+2 \mu)\left(\frac{\partial^{2} \Phi}{\partial x^{2}}+\frac{\partial^{2} \Phi}{\partial z^{2}}\right)+\left(\lambda^{\prime}+2 \mu^{\prime}\right) \frac{\partial}{\partial t}\left(\frac{\partial^{2} \Phi}{\partial x^{2}}+\frac{\partial^{2} \Phi}{\partial z^{2}}\right) \\
\rho \frac{\partial^{2} \Psi_{k}}{\partial t^{2}}=\mu\left(\frac{\partial^{2} \Psi_{k}}{\partial x^{2}}+\frac{\partial^{2} \Psi_{k}}{\partial z^{2}}\right)+\mu^{\prime} \frac{\partial}{\partial t}\left(\frac{\partial^{2} \Psi_{k}}{\partial x^{2}}+\frac{\partial^{2} \Psi_{k}}{\partial z^{2}}\right)
\end{array},\right.
$$

where the scalar potential function is $\Phi=\Phi(x, z, t)$, the vector potential function $\Psi_{k}$ is taken as $\Psi_{y}(x, z, t)$. From Equation (6) it is easy to find that $\Phi$ and $\Psi_{y}$ satisfy the same form of equation, so they should have the same form of expression. To begin with we try to find the solution in the form bellow:

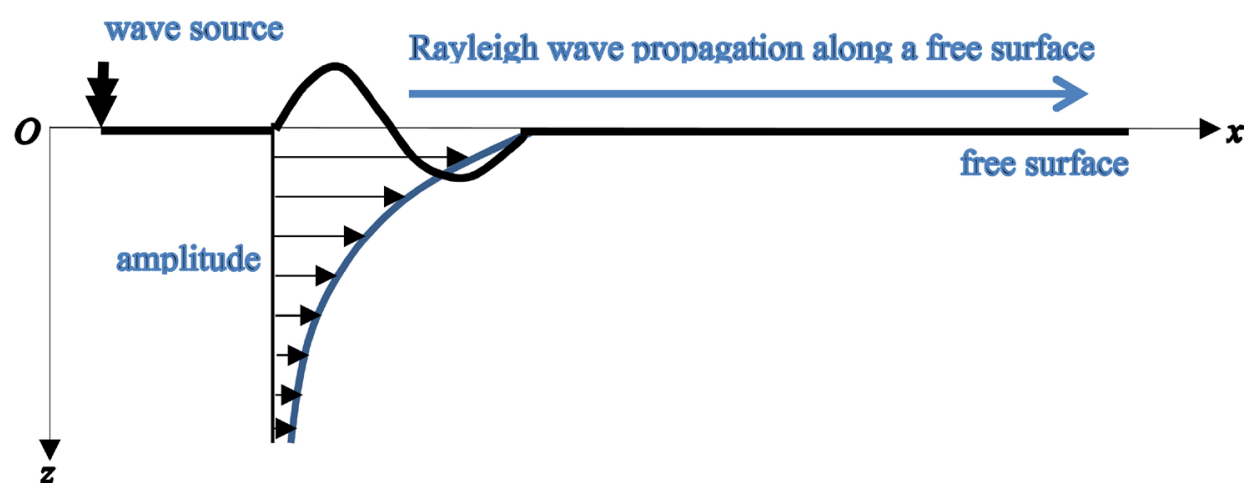

Figure 1. Diagram of Rayleigh wave. 


$$
\left\{\begin{array}{l}
\Phi(x, z, t)=a \mathrm{e}^{-\alpha z} \mathrm{e}^{i(K x-\omega t)} \\
\Psi_{y}(x, z, t)=b \mathrm{e}^{-\beta z} \mathrm{e}^{i(K x-\omega t)},
\end{array}\right.
$$

where $a$ and $b$ are both real non-zero constants; $\alpha$ and $\beta$ are positive real constants just like the condition in elastic medium, expressing the attenuations on the direction of axis $Z, \omega$ denotes the circular frequency of the motivation source. What is different here from that in the elastic condition is, $K$ denotes the complex circular wavenumber, whose real part is associated with the phase velocity of Rayleigh wave, and whose imaginary part reflects the attenuation effect of viscoelastic medium on the propagation along the free surface.

It is worth mentioning that $\alpha$ and $\beta$ above act as coefficients attenuating exponentially with the increase of depth in medium (both in elastic and viscoelastic condition), which were firstly introduced by Rayleigh [1] in elastic medium and is commonly accepted up to now. Therefore, the unknown variable in (7), namely the complex wavenumber $K$, is the key factor to determine the character of Rayleigh surface wave in Kelvin viscoelastic medium. In order to determine $K$, the boundary stress condition must be used

$$
\left\{\begin{array}{l}
\left.\sigma_{z x}\right|_{z=0}=0 \\
\left.\sigma_{z z}\right|_{z=0}=0
\end{array} .\right.
$$

Taking use of constitutive equation of Kelvin viscoelastic medium, the stress $\sigma_{z x}, \sigma_{z z}$ are expressed by displacement $u_{x}, u_{z}$. Finally, substitute the potential functions above with it

$$
\left\{\begin{array}{l}
u_{x}=\frac{\partial \Phi}{\partial x}-\frac{\partial \Psi_{y}}{\partial z} \\
u_{z}=\frac{\partial \Phi}{\partial z}+\frac{\partial \Psi_{y}}{\partial x}
\end{array}\right.
$$

Then

$$
\left\{\begin{array}{l}
\sigma_{z x}=\left(\mu+\mu^{\prime} \frac{\partial}{\partial t}\right)\left(\frac{\partial^{2} \Psi y}{\partial x^{2}}-\frac{\partial^{2} \Psi}{\partial z^{2}}+2 \frac{\partial^{2} \Phi}{\partial x \partial z}\right) \\
\sigma_{z z}=\left[(\lambda+2 \mu)+\left(\lambda^{\prime}+2 \mu^{\prime}\right) \frac{\partial}{\partial t}\right] \frac{\partial^{2} \Phi}{\partial z^{2}}+\left(\lambda+\lambda^{\prime} \frac{\partial}{\partial t}\right) \frac{\partial^{2} \Phi}{\partial x^{2}}+2\left(\mu+\mu^{\prime} \frac{\partial}{\partial t}\right) \frac{\partial^{2} \Psi_{y}}{\partial x \partial z}
\end{array} .\right.
$$

Substitute (10) with (7) and simplify it, on the free surface $z=0$ it satisfies

$$
\left\{\begin{array}{l}
\left.\sigma_{z x}\right|_{z=0}=\left\{-2 i \alpha K\left(\mu-i \omega \mu^{\prime}\right) \cdot a-\left[\mu\left(K^{2}+\beta^{2}\right)-i \omega \mu^{\prime}\left(K^{2}+\beta^{2}\right)\right] \cdot b\right\} \mathrm{e}^{i(K x-\omega t)} \\
\left.\sigma_{z z}\right|_{z=0}=\left\{\left\{\left[(\lambda+2 \mu) \alpha^{2}-\lambda K^{2}\right]-i \omega\left[\left(\lambda^{\prime}+2 \mu^{\prime}\right) \alpha^{2}-\lambda^{\prime} K^{2}\right]\right\} \cdot a-2 i \beta K\left(\mu-i \omega \mu^{\prime}\right) \cdot b\right\} \mathrm{e}^{i(K x-\omega t)} \cdot .
\end{array}\right.
$$

Substitute (8) with (11), we get the homogeneous linear equations for $a$ and $b$

$$
\left\{\begin{array}{l}
-2 i \alpha K\left(\mu-i \omega \mu^{\prime}\right) \cdot a-\left[\mu\left(K^{2}+\beta^{2}\right)-i \omega \mu^{\prime}\left(K^{2}+\beta^{2}\right)\right] \cdot b=0 \\
\left\{\left[(\lambda+2 \mu) \alpha^{2}-\lambda K^{2}\right]-i \omega\left[\left(\lambda^{\prime}+2 \mu^{\prime}\right) \alpha^{2}-\lambda^{\prime} K^{2}\right]\right\} \cdot a-2 i \beta K\left(\mu-i \omega \mu^{\prime}\right) \cdot b=0
\end{array} .\right.
$$

If there is non-zero root for (12), the coefficient determinant should be zero. 
Rearrange it

$$
\begin{aligned}
& \left(i \omega \mu^{\prime}-\mu\right)\left(\beta^{2}+K^{2}\right)\left\{\left[(\lambda+2 \mu) \alpha^{2}-\lambda K^{2}\right]-i \omega\left[\left(\lambda^{\prime}+2 \mu^{\prime}\right) \alpha^{2}-\lambda^{\prime} K^{2}\right]\right\} \\
& -4\left(i \mu+\omega \mu^{\prime}\right)^{2} \alpha \beta K^{2}=0
\end{aligned}
$$

The above Equation (13) is the characteristic equation to determine the complex wave number $K$ of Rayleigh surface wave. It is seen that for this problem, variable $K$ is not only related to the material modulus $\lambda, \mu, \lambda^{\prime}, \mu^{\prime}$, but also related to the circular frequency $\omega$. Meanwhile, it has mutual inference with $\alpha, \beta$.

In the special case of elastic medium, where $\lambda^{\prime}=\mu^{\prime}=0$ the imaginary part of complex wavenumber $K$ is zero. So, in such a case $K$ is exactly the circular wavenumber $k_{R}$ of Rayleigh wave in elastic medium. What has been known is that $\alpha^{2}=k_{R}^{2}-k_{P}^{2}, \beta^{2}=k_{R}^{2}-k_{S}^{2}$, here $k_{P}, k_{S}$ are respectively the circular wave numbers of elastic longitudinal wave and transverse wave. The equation of Kelvin viscoelastic Rayleigh wave (13) retrogrades to

$$
\left(2 k_{R}^{2}-k_{S}^{2}\right)^{2}-4 k_{R}^{2} \sqrt{\left(k_{R}^{2}-k_{P}^{2}\right)\left(k_{R}^{2}-k_{S}^{2}\right)}=0 .
$$

Equation (14) above is well-known equation for Rayleigh wave in elastic medium.

The solution of complex wavenumber $K$ can be obtained by solving characteristic equation of Kelvin viscoelastic Rayleigh wave (13). Obviously, except in elastic medium, it is commonly complicated to find out an analytic solution of complex wave number $K$.

\section{Simplified Form for Analysis of the Uniqueness Problem}

In this section, we try to get simpler form of the above Equation (13). We introduce two new complex moduli

$$
\Lambda=\lambda-i \omega \lambda^{\prime}, \quad \mathrm{M}=\mu-i \omega \mu^{\prime} .
$$

The introduced complex moduli $\Lambda, \mathrm{M}$ are composed of two parts, real parts of them are the Lame elastic coefficient $\lambda, \mu$, while imaginary parts act as the viscous part $\lambda^{\prime}, \mu^{\prime}$ of Lame coefficient times circular frequency $\omega$. Then Equation (13) can be rearranged into the following form:

$$
\Lambda K^{4}+\left[\Lambda \beta^{2}-(\Lambda+2 \mathrm{M}) \alpha^{2}+4 \mathrm{M} \alpha \beta\right] K^{2}-\alpha^{2} \beta^{2}(\Lambda+2 \mathrm{M})=0 .
$$

In the deduction above, we continue using of $\alpha, \beta$ as undetermined coefficients, but on complex field. Similar relationships with elastic case can be obtained by substituting Equation (7) into Equation (6) as bellow:

$$
\left\{\begin{array}{l}
\alpha^{2}=K^{2}-K_{P}^{2} \\
\beta^{2}=K^{2}-K_{S}^{2}
\end{array},\right.
$$

where $K_{P}^{2}=\frac{\rho \omega^{2}}{\Lambda+2 \mathrm{M}}, K_{S}^{2}=\frac{\rho \omega^{2}}{M} \cdot K_{P}, K_{S}$ respectively express wave numbers of longitudinal wave and transverse wave in viscoelastic medium, and like Ray- 
leigh wave number $K, K_{P}, K_{S}$ are complex numbers. Substitute (16) into (15), the following simplified equation can be obtained

$$
\left(2 K^{2}-K_{S}^{2}\right)^{2}-4 K^{2} \sqrt{\left(K^{2}-K_{P}^{2}\right)\left(K^{2}-K_{S}^{2}\right)}=0,
$$

which has the same form as the characteristic Equation (14) of Rayleigh wave for elastic medium except that the relevant parameters are on complex field. Rearrange the equation above, we can get a sixth-order algebraic equation including only even order terms on variable $K$, which is actually equivalent to a third-order algebraic equation about $K^{2}$

$$
16\left(K_{S}^{2}-K_{P}^{2}\right) K^{6}+\left(16 K_{P}^{2} K_{S}^{2}-24 K_{S}^{4}\right) K^{4}+8 K_{S}^{6} K^{2}-K_{S}^{8}=0 .
$$

Thus, as a result of the above simplification, we have derived the Rayleigh wave Equation (18) in Kelvin viscoelastic medium, a similar form to the one in elastic medium. Then according to the basic algebraic equation theory, there are three pairs of complex roots with inter conjugate. We can simply focus on three complex roots whose imaginary parts are positive as wave energy will not increase by itself. Certainly, we can use the Equation (18) to analyze the number of Rayleigh wave in Kelvin viscoelastic medium. However, the direct expression of the solution of the Equation (18) can be presumed to be rather cumbersome and difficult to analyze.

From the viewpoint of natural phenomena, Rayleigh wave is generated by interference between longitudinal wave and transverse wave, so in physical sense, the velocity (phase velocity) of Rayleigh wave should not surpass the velocity of transverse wave. Because the phase velocity of Rayleigh wave can be decided by $v=\frac{\omega}{\operatorname{Re}(K)}$, the phase velocity of transverse wave is decided by $v_{S}=\frac{\omega}{\operatorname{Re}\left(K_{S}\right)}$. The Rayleigh wave velocity is lower than that of transverse wave, that is:

$$
\operatorname{Re}(K)>\operatorname{Re}\left(K_{S}\right) .
$$

In this study, we proposal the Equation (18) combined with the above condition (19) to study the uniqueness problem of Rayleigh wave in half-space Kelvin viscoelastic medium. We speculate that there is only one root that satisfies the Rayleigh wave Equation (18) with the condition of $\operatorname{Re}(K)>\operatorname{Re}\left(K_{S}\right)$. This also means that there is only one possible characteristic root of the Equation (18), corresponding to the physical nature that the velocity of Rayleigh wave should be lower than the transverse wave.

The study of Currie et al. [6] [7] [8] in 1977 claims that there is not only one valid Rayleigh wave in linear viscoelastic half-space medium as well as the study of Chirita et al. [17] in 2014 on the method of solving several solutions of the Rayleigh wave in Kelvin viscoelastic condition. When they were studying the characteristic roots of Rayleigh wave, they also required that the characteristic roots should satisfy the characteristics of attenuation and the propagation direction, but none of them have taken consideration of the relative value between the velocity of Rayleigh wave and transverse wave. Take the example of the article of 
Chirita et al. [17], there is only one root in the paper whose corresponding Rayleigh wave velocity is lower than that of transverse wave, the ratio modulus of the other two roots to the velocity of transverse wave are larger than 2 , this means that the velocities of Rayleigh wave are far higher than that of transverse wave. Therefore, in physical sense, it is reasonable to presume that there is only one valid Rayleigh wave in their calculation examples.

\section{Examples of Mathematical Models with Plural Solutions}

In this section, we introduce examples of mathematical models representing phenomena, which allow plural solutions and whose solution would be unique under a condition in view of the natural phenomena, which shall be strongly connected with our proposal of the main problem in the next section.

The first example is the uniqueness of the diffusion equation in the infinite rod. Consider the following initial value problem for the diffusion equation.

Problem 1: Solve the following initial value problem

$$
\left\{\begin{array}{lc}
\frac{\partial u}{\partial t}(t, x)=\frac{\partial^{2} u}{\partial x^{2}}(t, x) & (t>0,-\infty<x<\infty) \\
u(0, x)=f(x) & (-\infty<x<\infty)
\end{array} .\right.
$$

We shall discuss unique solvability of (20). Since the equation is linear, what we have to study is the following problem.

Problem 2: Assume that $u \in C_{t}^{1}[0, \infty) \times C_{x}^{2}(\mathbb{R})$ satisfies

$$
\left\{\begin{array}{lc}
\frac{\partial u}{\partial t}(t, x)=\frac{\partial^{2} u}{\partial x^{2}}(t, x) & (t>0,-\infty<x<\infty) \\
u(0, x) \equiv 0 & (-\infty<x<\infty)
\end{array} .\right.
$$

In this case, does it hold that $u \equiv 0$ ?

In general, the answer to Problem 2 is negative. There is a following famous counterexample.

Example 1:

$$
u(x, t)=\sum_{n=0}^{\infty} f^{(n)}(t) \frac{x^{2 n}}{(2 n) !}, \quad f(t)=\left\{\begin{array}{ll}
\mathrm{e}^{-1 / t^{2}} & (t>0) \\
0 & (t \leq 0)
\end{array} .\right.
$$

The function constructed in (22) is known to be convergent and satisfies (21), but it is not identically zero. Confer [18], for this example. This example grows faster than $\mathrm{e}^{a|x|^{2}}$ for any $a>0$, which cannot be the case in the real phenomena, for example, consider the diffusion of the heat. If we preclude such unrealistic examples, then the unique solvability of the initial problem (20) holds.

Theorem 1 (Unique solvability of the initial value for the diffusion equation):

Assume that $u(x, t) \in C([0, \infty) \times \mathbb{R}) \cap C^{2}((0, \infty) \times \mathbb{R})$ satisfies $(21)$ and there exist some $C>0$ and $a>0$ such that

$$
|u(x, t)| \leq C \mathrm{e}^{a|x|^{2}}, \text { for } \forall x<\mathbb{R} .
$$

Then $u(x, t)$ identically vanishes. 
This theorem is also proved in [18]. In fact, there are more advanced researches in Problem 2, for which confer [19] and [20].

Next, let us study two uniqueness problems relating the computerized tomography ("CT" for short). It is known that the mathematical problem of CT is formulated as follows.

Problem 3: Reconstruct the function $f(x, y)$ defined on the two-dimensional space $\mathbb{R}^{2}$ from the data

$$
\left\{\int_{l} f(x, y) \mathrm{d} l \mid l \text { is a line in } \mathbb{R}^{2}\right\} .
$$

In general, for a function $f(x)$ defined for $x$ in $\mathbb{R}^{n}$, its Radon transform is defined by:

$$
R f(H(\theta, s)) \equiv R f(\theta, s):=\int_{\theta^{\perp}} f(s \theta+y) \mathrm{d} y,
$$

where $\theta \in S^{n-1}, \quad s \in \mathbb{R}, \theta^{\perp}:=\left\{x \in \mathbb{R}^{n} ; x \perp \theta\right\}, y \in \theta^{\perp}$.

Note that the pair $(\theta, s)$ is identified with the hyperplane

$$
H=H(\theta, s)=\left\{x \in \mathbb{R}^{n} ; x \cdot \theta=s\right\},
$$

and that $H(\theta, s)=H(-\theta,-s)$.

We also note that the function $f(x, y)$ represents the density of the human body at the point $(x, y)$ in $\mathbb{R}^{2}$, where the section of the human body by a plane is of interest. Confer [21] for the mathematical problems in CT.

Let us study the uniqueness of the exterior problem of the Radon transform. The exterior problem of the Radon is formulated by the following.

Problem 4: Let $\boldsymbol{K}$ be a compact convex set in $\mathbb{R}^{n}$ and $f \in C\left(\mathbb{R}^{n} \backslash \boldsymbol{K}\right)$. Assume that for any hyperplane $\xi$ with $\xi \cap \boldsymbol{K}=\boldsymbol{\varnothing}$, its Radon transform $R f(\xi)$ absolutely converges. Reconstruct $\{f(x) \mid x \notin \boldsymbol{K}\}$ out of the data $\{R f(\xi) \mid \xi \cap \boldsymbol{K}=\boldsymbol{\varnothing}\}$.

Radon transform being linear, its uniqueness problem leads as.

Problem 5: Let $\boldsymbol{K}$ be a compact convex set in $\mathbb{R}^{n}$ and $f \in C\left(\mathbb{R}^{n} \backslash \boldsymbol{K}\right)$. Assume that for any hyperplane $\xi$ with $\xi \cap \boldsymbol{K}=\boldsymbol{\varnothing}$, its Radon transform $R f(\xi)$ absolutely converges. Does the condition $R f(\xi)=0$ for any $\xi \cap \boldsymbol{K}=\boldsymbol{\varnothing}$ imply that $f(x)=0$ for $x \notin \boldsymbol{K}$ ?

The answer to this problem is not affirmative in general.

Example 2: For $n=2$ and $1<m \in \mathbb{N}$, consider

$$
f\left(x_{1}, x_{2}\right):=\frac{1}{\left(x_{1}+i x_{2}\right)^{m}} .
$$

The integral of $f\left(x_{1}, x_{2}\right)$ along any line not passing through the origin converges to 0 by Cauchy integral theorem, which leads that the uniqueness of the exterior problem would not hold by lettering $\boldsymbol{K}$, for example, $\boldsymbol{K}=\left\{x_{1}^{2}+x_{2}^{2} \leq 1\right\}$. In this example, the support the function $f$ is the whole plane. It cannot happen in the problem of CT since any section of a human body by a plane must be boundedly supported for there is no human being who occupies the unbounded area or space. Mathematically, the following theorem holds. 
Theorem 2 (Uniqueness of the exterior problem for the Radon transform):

Let $\boldsymbol{K}$ be a compact convex set in $\mathbb{R}^{n}$ and $f \in C\left(\mathbb{R}^{n} \backslash \boldsymbol{K}\right)$. Assume that $R f(\xi)=0$ for $\xi \cap \boldsymbol{K}=\boldsymbol{\varnothing}$ and that

$$
|x|^{k} f(x) \rightarrow 0 \text { as }|x| \rightarrow \infty \text {, for } \forall k \in \mathbb{N} .
$$

Then $f(x)=0$ for $x \notin \boldsymbol{K}$.

For the proof of this theorem and the example (26), confer [23]. It is known that this theorem holds for the most singular functions, the hyperfunctions, which was proved by Takiguchi and Kaneko [24]. Therefore, it is essential that the condition (27) is satisfied with the uniqueness of the exterior problem to hold, and the regularity of the function would not matter at all to this problem. Note also that by the condition (27), all unrealistic examples like (26) constructed in Example 2, which break the uniqueness, are excluded.

Finally let us study the global uniqueness problem for the Radon transform, which reads as.

Problem 6: Let a function $f$ be defined on $\mathbb{R}^{n}$. Assume that for any hyperplane $\xi$, its Radon transform $R f(\xi)$ converges absolutely and equals to 0 . Does this condition imply that $f \equiv 0$ ?

The answer to this problem is known to be negative without any global growth condition, for which confer [25] and [26]. The counterexamples constructed in [25] and [26] are very artificial functions. In both papers, we regard that $\mathbb{R}^{2} \cong \mathbb{C}$ and let $z=x+i y$ for a complex number $z$, where $x=\operatorname{Re} z$ and $y=\operatorname{Im} z$. The one constructed in [25] grows super-exponentially in $\left\{\frac{1}{x}<y<\frac{2}{x}\right\}$ as $x \rightarrow \infty$. The other one also grows super-exponentially in $\left\{\frac{1}{4}<x^{2}-y^{2}<4\right\}$ as $x \rightarrow \infty$. In some inverse problems in quantum mechanics, we obtain the observation data are obtained as the Radon transform (or the X-ray transform) of the potential (cf. [27]), however, the usual potentials would not grow super-exponentially, in view of which, the counterexamples constructed in [25] and [26] are unrealistic. If we assume that $f \in L^{1}\left(\mathbb{R}^{n}\right)$ then the global uniqueness holds.

Theorem 3 (Global uniqueness for the Radon transform):

Let $f \in L^{1}\left(\mathbb{R}^{n}\right)$. Then $R f(\xi) \equiv 0$ implies that $f \equiv 0$.

The proof of Theorem 3 is in [25] and is too simple to omit.

We first note that for a fixed $\theta \in S^{n-1}, R f(\theta, s)$ is well-defined for almost all $s \in \mathbb{R}$ and $R f(\theta, s) \in L^{1}(\mathbb{R})$ as a function of $s \in \mathbb{R}$. Denote $x=\left(x^{\prime}, x_{n}\right)$, $x^{\prime} \in \mathbb{R}^{n-1}, \quad x_{n} \in \mathbb{R}$, then we have

$$
\hat{f}\left(0, \xi_{n}\right)=\int_{\mathbb{R}} \mathrm{e}^{-i x_{n} \xi_{x}} \mathrm{~d} x_{n} \int_{\mathbb{R}^{n-1}} f\left(x^{\prime}, x_{n}\right) \mathrm{d} x^{\prime}=0,
$$

where for $\xi \in \mathbb{R}^{n}$,

$$
\hat{f}(\xi)=\int_{\mathbb{R}^{n}} \mathrm{e}^{-i x \cdot \xi} f(x) \mathrm{d} x,
$$

is the Fourier transform of the function $f$. In the same way, we can prove that for any $\theta \in S^{n-1}$, the condition $R f(H)=0$ for any hyperplane $H \perp \theta$ implies 
$\hat{f}(r \theta)=0$ for any $r \in \mathbb{R}$. Since $\hat{f}$ is continuous by Riemann-Lebesgue theorem, we conclude that $\hat{f} \equiv 0$. Therefore $f \equiv 0$.

Note that this theorem holds under a much weaker condition.

Theorem 4 (Extension of the global uniqueness for the Radon transform):

Let a measurable function $f$ defined on $\mathbb{R}^{n}$ satisfy that for any $\varepsilon>0$ there exists a constant $C_{\varepsilon}>0$ such that

$$
|f(x)| \leq C_{\varepsilon} \mathrm{e}^{\varepsilon|x|} \text { for almost every } x \in \mathbb{R}^{n} .
$$

Then $R f(\xi) \equiv 0$ implies that $f \equiv 0$.

The theorem is proved by interpreting the proof Theorem 3, in terms of the Fourier hyperfunctions. In view of practical applications, the condition that $f \in L^{1}\left(\mathbb{R}^{n}\right)$ may not be sufficient, especially in view of the potentials in quantum mechanics. In some inverse scattering problem in quantum mechanics, we have to reconstruct the potential from its Radon (or X-ray) transform, confer the paper [27] [28] for this kind of inverse problems. The short-range potentials satisfying the condition that $f \in L^{1}\left(\mathbb{R}^{n}\right)$, the long-range ones decay much slower, that is

$$
f(x) \rightarrow 0 \text { as }|x| \rightarrow 0
$$

which is included in the condition (28).

In this section, we have introduced three uniqueness problems where the uniqueness would hold without any conditions, which are uniquely solvable under natural conditions in view of the real phenomena. At the end of this section, we summarize our claim in view of these three examples.

In the modeling process of phenomena, we need to idealize and simplify them, which results in that there are numbers of solutions to mathematical models representing the phenomena. If there is a natural assumption in accordance with the phenomenon, then it makes the mathematical model for the phenomenon has the unique solution representing the phenomenon itself.

\section{Conclusion and Discussion}

We derived the characteristic equation of Rayleigh surface wave in Kelvin viscoelastic half-space in a brief way and in terms of complex wave number. We then proposed a relatively convenient and concise method which is suitable for analyzing the validity of characteristic roots in a physical sense and presumed that there is only one Rayleigh wave in Kelvin viscoelastic half-space surface. We introduced uniqueness problems where the uniqueness would hold without any conditions, which are uniquely solvable under natural conditions in view of the real phenomenon. We conjecture that the essence of the unique problem for Rayleigh waves is identical.

Conjecture: There being researches show that there are plural Rayleigh waves in Kelvin viscoelastic medium. If we assume a natural condition in accordance with the natural phenomenon and the medium, then the Rayleigh wave must be unique. 
By "a natural condition" in this conjecture, we mean the condition that the speed of the Rayleigh wave would not exceed the transverse one. Since the Rayleigh wave is generated by the interference of longitudinal wave and transverse wave, it is very natural that the speed of Rayleigh wave is slower than longitudinal and transverse ones. The authors claim that the uniqueness of the Rayleigh wave would be proved under this condition, whose story is similar to how the study of the eigensolutions of crack-tip stress singularity in elastic media has been developed [29].

In the future, we may have to develop the theory much further. In the study of Problem 2, uniqueness is proved under a more general assumption than the natural one (cf. [20]). It is also the same for Problem 5; the condition (27) is more general than the condition that the function $f$ is compactly supported which is very natural in view of CT. In Theorem 4, a solution to Problem 6, a much more general condition than the natural one, $(28)$ is given. The condition (28) allows the growth of the function which is far from the estimate (29) of the long-range potentials.

In the study of the uniqueness of the Rayleigh wave on viscoelastic media, similar development can be expected; we firstly study the uniqueness under the condition that its speed is slower than the transverse one, which is a natural condition in view of the phenomenon. Then after proving it, we may have to study how we can weaken this condition.

\section{Acknowledgements}

This work was supported by Shanghai Committee of Science and Technology of China (17ZR1402800).

\section{Conflicts of Interest}

The authors declare no conflicts of interest regarding the publication of this paper.

\section{References}

[1] Rayleigh, L. (1885) On Waves Propagated along the Plane Surface of an Elastic Solid. Proceedings of the London Mathematical Society, 17, 4-11. https://doi.org/10.1112/plms/s1-17.1.4

[2] Thomson, W.T. (1950) Transmission of Elastic Waves through a Stratified Solid Medium. Journal of Applied Physics, 21, 89-93. https://doi.org/10.1063/1.1699629

[3] Haskell, N.A. (1953) The Dispersion of Surface Waves on Multilayered Media. Bulletin of the Seismological Society of America, 43, 17-34.

[4] Scholte, J.G. (1947) On Rayleigh Waves in Visco-Elastic Media. Physica, 13, 245-250. https://doi.org/10.1016/0031-8914(47)90083-9

[5] Bland, D.R. (1960) The Theory of Linear Viscoelasticity. Pergamon, Oxford.

[6] Currie, P.K., Hayes, M.A. and O'Leary, P.M. (1977) Viscoelastic Rayleigh Waves. Quarterly of Applied Mathematics, 35, 35-53. https://doi.org/10.1090/qam/99648

[7] Currie, P.K. and O'Leary, P.M. (1978) Viscoelastic Rayleigh Waves II. Quarterly of 
Applied Mathematics, 36, 445-454. https://doi.org/10.1090/qam/99642

[8] Currie, P.K. (1979) Viscoelastic Surface Waves on a Standard Linear Solid. Quarterly of Applied Mathematics, 37, 332-336. https://doi.org/10.1090/qam/99632

[9] Romeo, M. (2001) Rayleigh Waves on a Viscoelastic Solid Half-Space. Journal of the Acoustical Society of America, 110, 59-67. https://doi.org/10.1121/1.1378347

[10] Romeo, M. (2002) Uniqueness of the Solution to the Secular Equation for Viscoelastic Surface Waves. Applied Mathematics Letters, 15, 649-653. https://doi.org/10.1016/S0893-9659(02)80019-2

[11] Nkemzi, D. (1997) A New Formula for the Velocity of Rayleigh Waves. Wave Motion, 26, 199-205. https://doi.org/10.1016/S0165-2125(97)00004-8

[12] Ivanov, T.P. and Savova, R. (2005) Viscoelastic Surface Waves of an Assigned Wavelength. European Journal of Mechanics-A/Solids, 24, 305-310. https://doi.org/10.1016/j.euromechsol.2004.11.002

[13] Ivanov, T.P. and Savova, R. (1993) Viscoelastic Surface Waves. European Journal of Mechanics-A/Solids, 12, 667-677.

[14] Ivanov, T.P. and Savova, R. (2014) Motion of the Particles Due to Viscoelastic Surface Waves of an Assigned Frequency. Mathematics and Mechanics of Solids, 19, 725-731. https://doi.org/10.1177/1081286513486473

[15] Carcione, J.M. and José, M. (1992) Rayleigh Waves in Isotropic Viscoelastic Media. Geophysical Journal International, 108, 453-464. https://doi.org/10.1111/j.1365-246X.1992.tb04628.x

[16] Savova, R. and Ivanov, T.P. (2007) On Viscous Effects in Surface Wave Propagation. Journal of Theoretical and Applied Mechanics, 35, 25-34.

[17] Chirita, S., Ciarletta, M. and Tibullo, V. (2014) Rayleigh Surface Waves on a Kelvin-Voigt Viscoelastic Half-Space. Journal of Elasticity, 115, 61-76. https://doi.org/10.1007/s10659-013-9447-0

[18] Widder, D.V. (1975) The Heat Equation. Academic Press, New York, San Francisco, London.

[19] Chung, S.Y. and Kim, D. (1994) An Example of Nonuniqueness of the Cauchy Problem for the Heat Equation. Communications in Partial Differential Equations, 19, 1257-1261. https://doi.org/10.1080/03605309408821054

[20] Chung, S.Y. (1999) Uniqueness in the Cauchy Problem for the Heat Equation. Proceedings of the Edinburgh Mathematical Society, 42, 455-468. https://doi.org/10.1017/S0013091500020459

[21] Natterer, F. (2001) The Mathematics of Computerized Tomography. SIAM, Philadelphia. https://doi.org/10.1137/1.9780898719284

[22] Phan-Thien, N. and Mai-Duy, N. (2017) Understanding Viscoelasticity: An Introduction to Rheology. 3rd Edition, Springer, Cham. https://doi.org/10.1007/978-3-319-62000-8

[23] Helgason, S. (1999) The Radon Transform. Second Edition, Birkhäuser, Boston, Basel, Berlin. https://doi.org/10.1007/978-1-4757-1463-0

[24] Takiguchi, T. and Kaneko, A. (1995) Radon Transform of Hyperfunctions and Support Theorem. Hokkaido Mathematical Journal, 24, 63-103. https://doi.org/10.14492/hokmj/1380892536

[25] Zalcman, L. (1982) Uniqueness and Nonuniqueness for the Radon Transform. Bulletin of the London Mathematical Society, 14, 241-245. https://doi.org/10.1112/blms/14.3.241

[26] Takiguchi, T. (2001) Remarks on Modification of Helgason's Support Theorem. II. 
Proceedings of the Japan Academy, Series A, Mathematical Sciences, 77, 87-91. https://doi.org/10.3792/pjaa.77.87

[27] Enss, V. and Weder, R. (1995) The Geometrical Approach to Multidimensional Inverse Scattering. Journal of Mathematical Physics, 36, 3902-3921. https://doi.org/10.1063/1.530937

[28] Takiguchi, T. (2000) An Inverse Problem for Free Channel Scattering. Inverse Problems and Related Topics (Kobe 1998), Chapman \& Hall/CRC, London, Research Notes in Mathematics Series 419, 165-179. https://doi.org/10.1201/9780429187841-13

[29] Williams, M.L. (1957) On the Stress Distribution at the Base of a Stationary Crack. Journal of Applied Mechanics, 24, 109-114. 